\title{
Dislodgement of the Fully Expanded Stent and the Management of This Complication by Using Crushing Technique
}

\author{
๑ Sara Çetin Şanlıalp1, ๑ Işık Tekin², ๑ Musa Şanlıalp2
}

${ }^{1}$ Servergazi State Hospital, Clinic of Cardiology, Denizli, Turkey

2Denizli State Hospital, Clinic of Cardiology, Denizli, Turkey

\begin{abstract}
Dislodgement of the coronary stent during percutaneous coronary intervention (PCI) is a rare and serious complication. This complication usually occurs when an undeployed stent unintentionally dislocates from the balloon. A few cases of fully expanded stent dislodgement have been reported in the literature. If not managed properly, it may cause major adverse events and even death.
\end{abstract}

\section{Introduction}

Percutaneous coronary intervention (PCI) is used in the treatment of coronary artery diseases due to its high success rate today ${ }^{(1)}$. In recent years, coronary angioplasty has been supported by stenting because of reducing the risk of revascularization and restenosis ${ }^{(2)}$. Although PCI has become a widespread and effective modality
We reported an unusual case of a 39-year-old male who presented with fully expended stent dislodgement in the mid right coronary artery following retrieving of deflated stent balloon during secondary PCI.

Keywords: Crushing technique, stent dislodgement, percutaneous coronary intervention today, procedural complications can still develop ${ }^{(3)}$. Stent dislodgement is a serious complication of PCI and its incidence has decreased with the use of improved equipments and modern stents ${ }^{(4)}$. This complication is usually associated with significant morbidity including emergency coronary artery bypass graft surgery, acute myocardial infarction (AMI) and systemic/coronary embolizations $^{(5)}$. Most of the previously reported stent

Address for Correspondence: Sara Çetin Şanlıalp, Servergazi State Hospital, Clinic of Cardiology, Denizli, Turkey

e-mail: saracetin@hotmail.com.tr ORCID: orcid.org/0000-0001-9328-9197

Received: 17.04.2020 Accepted: 27.07.2020

Cite this article as: Çetin Şanlıalp S, Tekin I, Şanlıalp M. Dislodgement of The Fully Expanded Stent and The Management of This Complication by Using Crushing Technique. EJCM 2020;8(3):157-162.

DOI: 10.32596/ejcm.galenos.2020.04.019

${ }^{\circ}$ Copyright 2020 by Heart and Health Foundation of Turkey (TÜSAV) / E Journal of Cardiovascular Medicine published by Galenos Publishing House. 
dislodgement cases have occurred before the complete expansion of the stent in the target vessel and it is almost impossible to remove the fully expanded stent ${ }^{(6)}$. We reported a case of fully expanded stent dislodgement after successful implantation in a patient with acute inferior STsegment elevation myocardial infarction (STEMI).

\section{Case Report}

A 39-year-old male patient was admitted to our emergency department with sudden onset of chest pain. He did not have any additional cardiac risk factors except smoking. He had a blood pressure of $110 / 70 \mathrm{mmHg}$, a heartbeat rate of $72 / \mathrm{min}$, and a fever of 36.3 degrees. The oxygen saturation measured in the pulse oximeter was $98 \%$ and his physical examination was normal. Electrocardiography (EKG) showed normal sinus rhythm with ST-segment elevations in inferior leads and reciprocal changes in anterior leads. An echocardiogram revealed mild hypokinesia at the inferior and posterior wall and the estimated left ventricular ejection fraction was $45 \%$. After using antithrombotic agents consisting of ticagrelor $180 \mathrm{mg}$, acetylsalicylic acid (aspirin) $100 \mathrm{mg}$ and subcutaneous low molecular weight heparin (LMWH), he underwent primary catheterization in 30 minutes after admission.

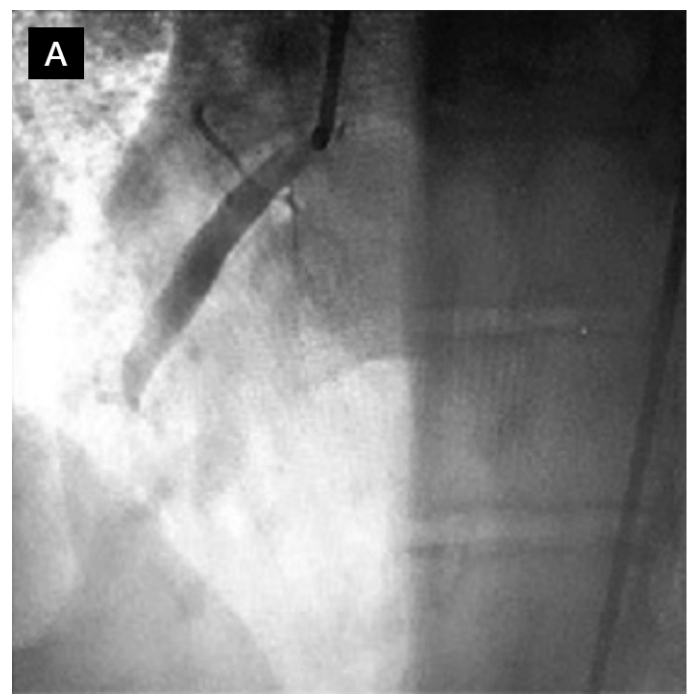

A diagnostic coronary angiography via right femoral approach revealed total occlusion with massive thrombus at the middle segment of right coronary artery (RCA) (Figure 1A). There was no significant stenosis in the left main coronary artery, left anterior descending artery and circumflex artery. PCI was planned for the significant lesion of the RCA. The right coronary ostium was engaged with a 6 Fr Judkins-Right catheter and 0.014-inch floppy guidewire (ChoICE, Boston Scientific, Minnesota, USA) was used to cross the lesion in the mid RCA. First, the lesion was pre-dilated with a 3.0x20 balloon and followed by a $4.0 \times 20 \mathrm{~mm}$ balloon (Simpass plus, Simeks Medical, İstanbul, Turkey). Then 4.0x $24 \mathrm{~mm}$ Rebel bare-metal stent (Boston Scientific MN, USA) was deployed. During this process, the thrombus in the lesion progressed to the distal RCA due to mechanical effect of pre-dilation (Figure 1B) so distal RCA was pre-dilated with a 3.0x12 mm balloon (Simpass plus, Simeks Medical, İstanbul, Turkey) and 2 vials of abciximab were injected by intracoronary route for dissolving thrombus. But, the thrombolysis in myocardial infarction (TIMI) III flow grade could not be supplied and an additional iv 2500 IU of unfractional heparin (UFH) was added to the initial iv 5000 IU of UFH before the termination of the procedure. The chest pain did not recur after PCI and his hemodynamic status was stable.

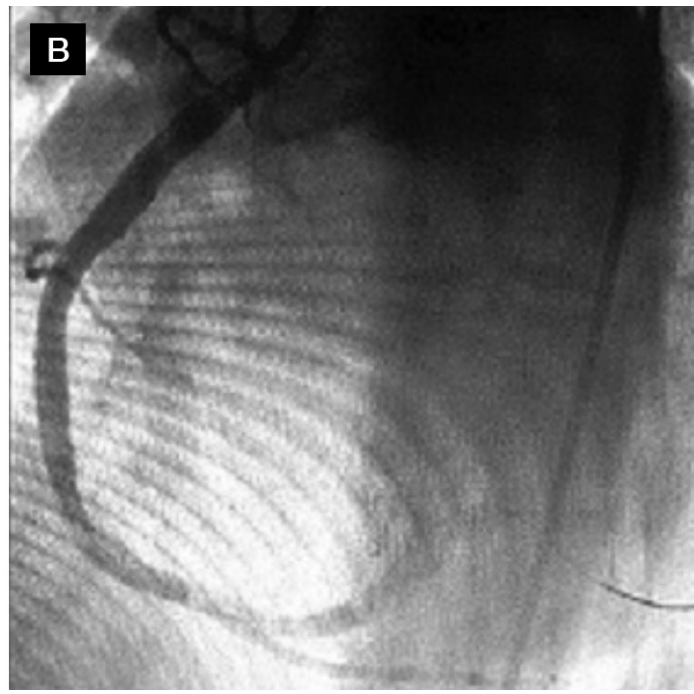

Figure 1. (A) A diagnostic coronary angiography showed total occlusion of the mid right coronary artery in the anterior-posterior cranial view. (B) The lesion with thrombus in the distal right coronary artery after stenting of mid right coronary artery in the anteriorposterior cranial view 
Then, low dose betablocker with subcutaneous LMWH was added to the standard antithrombotic treatment. Because of the persistence of the ST-segment elevations in the inferior leads on EKG and high plasma troponin levels, the secondary PCI was planned next day for the same coronary vessel. After pre-dilation with the $1.5 \times 10$ and 2.0x20 mm balloons (Simpass plus, Simeks Medical, İstanbul, Turkey), a 3.0x20 mm Promus drug-eluting stent (Boston Scientific, California, USA) was delivered through a previous stent (Figure 2A) and successfully implanted in the thrombotic distal lesion (Figure 2B, 2C).
Surprisingly, the fully expanded stent in the mid RCA was dislodged and deformed during retrieving of the deflated stent balloon (Figure 2D, 3A). Complete fully expanded stent dislodgement is a very rare case after successful implantation. There was no perforation or dissection in the coronary images after stent dislodgement. We planned to use the twisted wire technique to retrieve the fully expanded stent because the first guide wire was in situ. However, this technique was discontinued due to catheterrelated vasospasm during the advancement of the second wire. Then, the crushing technique was used urgently due
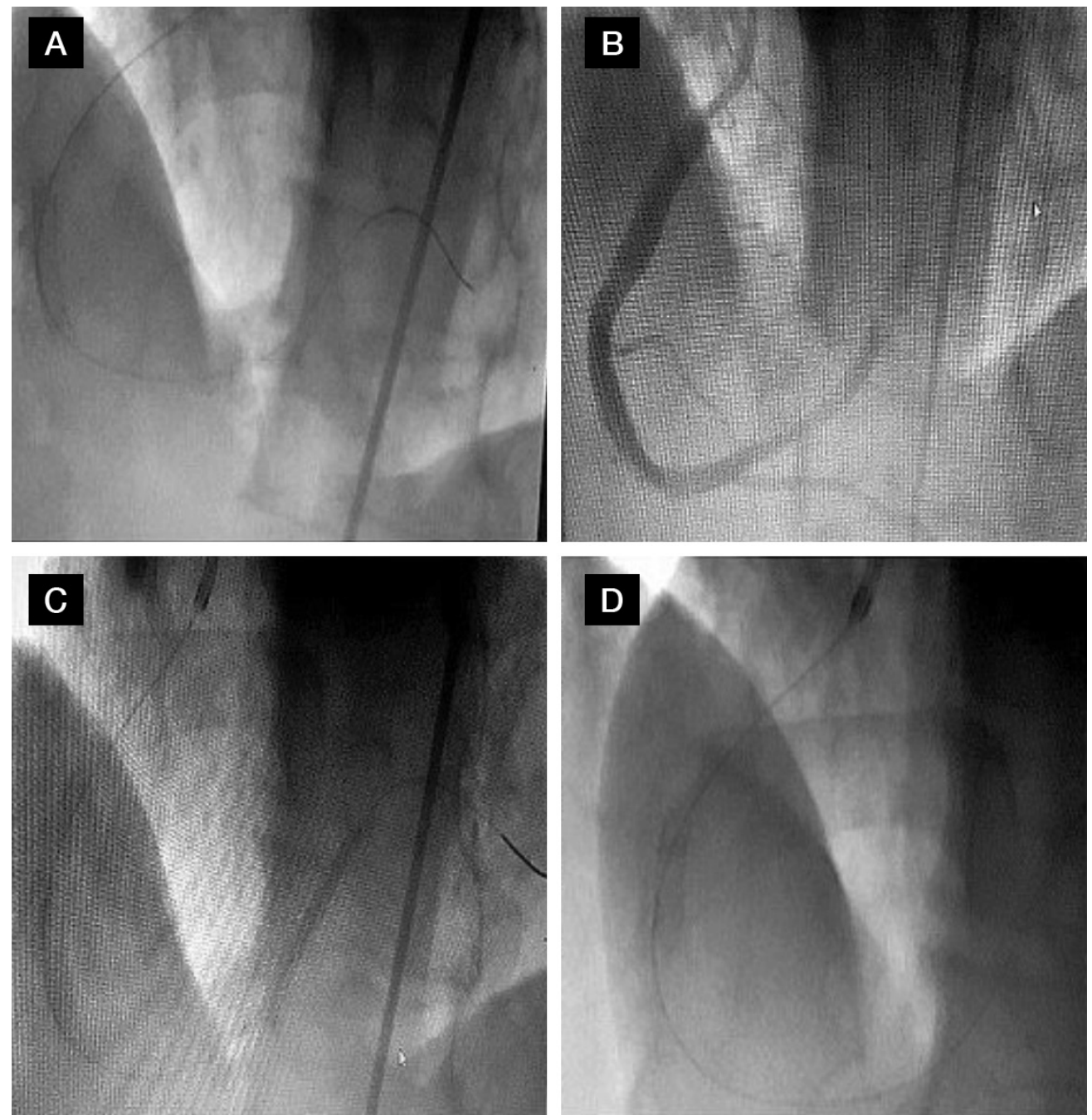

Figure 2. (A) Image of stent deployment in the mid right coronary artery (cranial anterior-posterior view) in angiography performed the following day. (B) Cranial anterior-posterior view of the unresolved thrombus in the distal right coronary artery. (C) Deployment of the drug-eluting stent in the distal lesion with thrombus in the cranial anterior-posterior view. (D) Dislodgement and deformation of the proximal fully expanded stent during the retrieval of the deflated balloon (cranial anterior-posterior view) 
to impossible removing and the risk of hemodynamic deterioration. The dislodged fully expanded stent was successfully mounted into coronary vessel with a $4.5 \times 28$ mm Rebel bare-metal stent (Boston Scientific MN, USA) and post-dilation was performed with a non-compliant 4.5x15 balloon (NC Trek, Abbott Vascular, Santa Clara, California) (Figure 3B, 3C). Finally, a second 3.5x12 $\mathrm{mm}$ Rebel bare-metal stent (Boston Scientific MN, USA) was deployed overlapping with the distal stent after postdilation of distal stent with a $3.5 \times 15$ balloon (Simpass plus, Simeks Medical, İstanbul, Turkey) (Figure 3D). After the
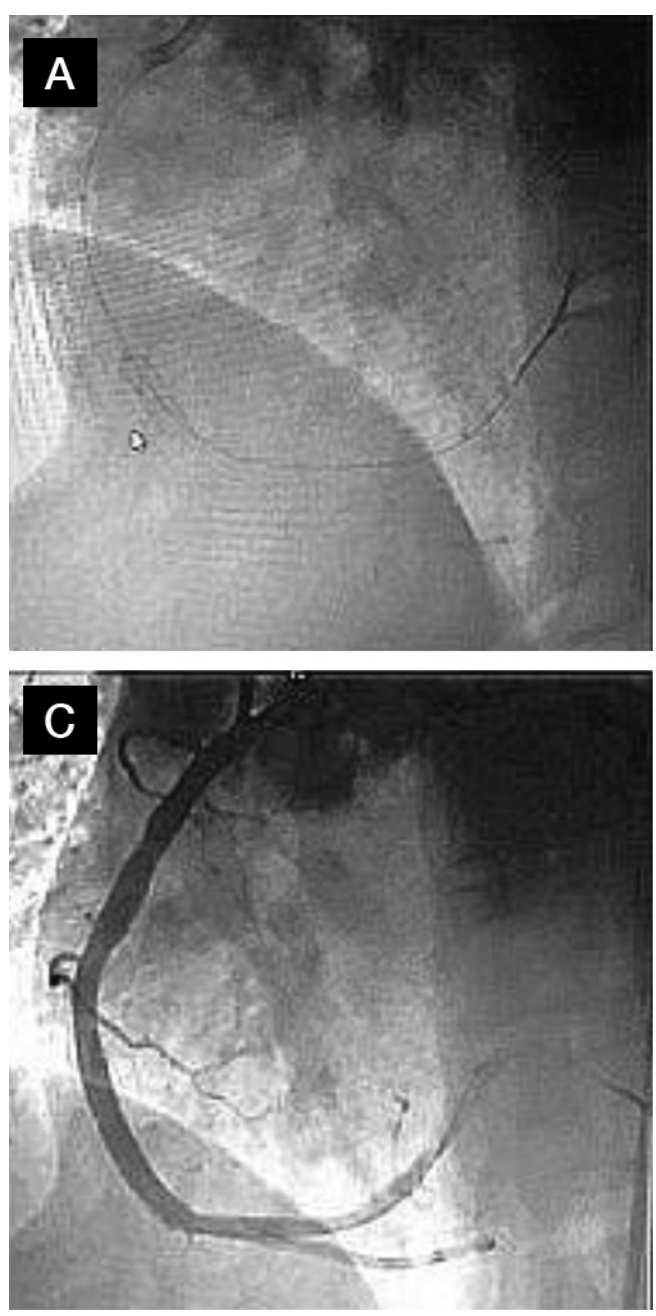

second PCI, the troponin levels lowered and ECG findings improved during follow-up in the coronary care unit and he was discharged in stable condition with appropriate advice and medicine including dual antithrombotic agents. Two months later, the control angiography was performed due to previous complicated PCI and there was no restenosis or complication.

\section{Discussion}

Stent dislodgement is a rare but serious complication of PCI and may cause arterial thrombosis, systemic and
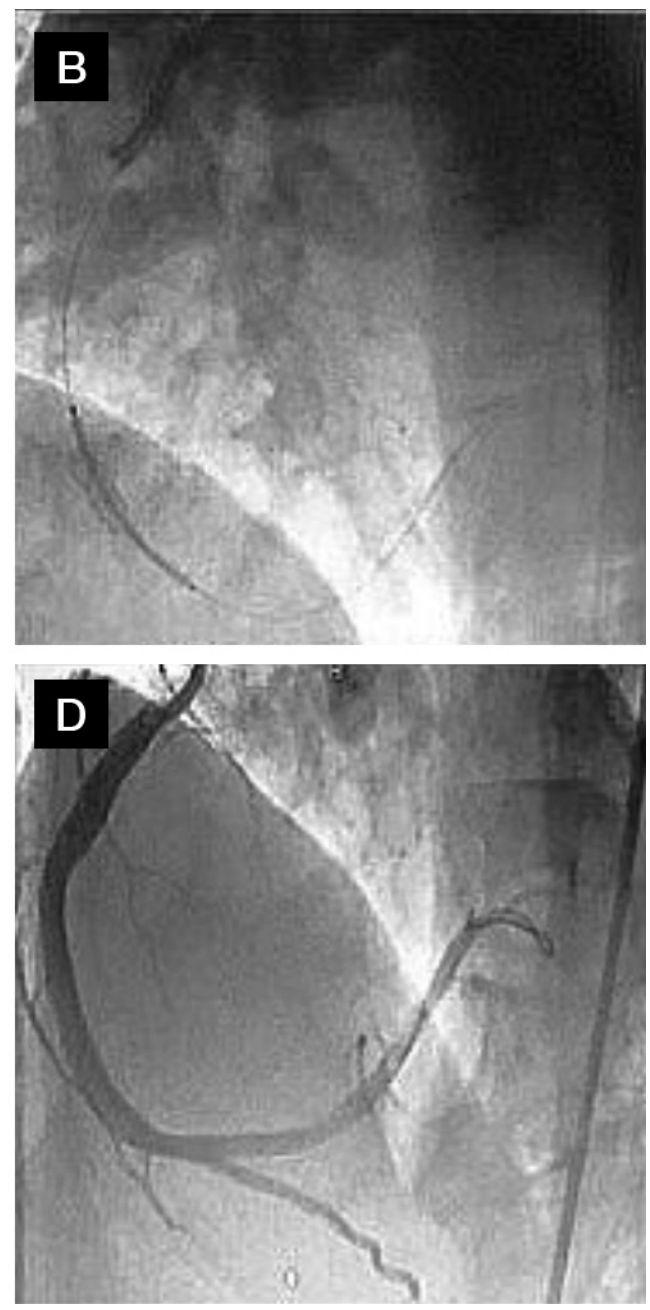

Figure 3. (A) The forcing of the proximal fully expanded stent into the catheter and advancing to the crux of right coronary artery (cranial anterior-posterior view). (B) Mounting of the dislodged stent in the coronary vessel successfully with another stent by using Crushing Technique (cranial anterior-posterior view). (C) Providing TIMI 3 flow grade in mid RCA after crushing technique (cranial anterior-posterior view). (D) In angiography, cranial anterior-posterior view of distal right coronary artery after stenting

TIMI: Thrombolysis in myocardial infarction, RCA: Right coronary artery 
coronary embolizations, adverse cardiovascular events such as acute cardiac myocardial infarction, stroke and even death ${ }^{(7)}$. Most of the cases develop due to undeployed stent stripped from the balloon. This mechanism usually occurs due to serious calcifications, angulated lesions, short small stents, unexpanded stents and manual handling of stent. Also, primarily stent implantation in the proximal segments rather than distal may lead to dislodgement ${ }^{(8,9)}$. Its incidence ranges from $0.3 \%$ to $8 \%$ due to pre-mounting technologies and modern equipments ${ }^{(10)}$.

In stent dislodgement, the retrieval of stent should be first choice. If not possible, it may be mounted into the coronary vessel using another stent by crushing technique or re-implanted in the appropriate segment ${ }^{(11)}$. The retrieving methods can be performed surgically or percutaneously. Percutaneous retrieval methods should be preferred if the patient's vital signs and clinical status are stable $^{(12)}$. Several retrieval methods are defined including biliary forceps, twisted guide wires, multipurpose baskets, snare and small-balloon technique. The most preferred method is the small balloon technique and has a success rate of $70 \%{ }^{(13)}$. However, the dislodgement of the fully expanded stent is very rare and has a high potential to cause catastrophic events ${ }^{(8)}$.

Although the migration mechanism of stent was elusive to understand, we suspected two factors including the underestimation of vessel diameter and the first stenting of proximal lesion rather than distal. However, the proximal culprit lesion was stented because of AMI in this case. We hoped that the thrombus in the distal RCA would be resolved with antithrombotic agents. But, we failed and had to stent the distal lesion. The use of intravascular ultrasound (IVUS) might determine the vessel diameter clearly and it might reveal large amounts of remnant plaque burden and insufficient plaque modification. However, we deployed the stent according to the estimated vessel diameter due to the absence of IVUS and the size of RCA was larger than the standard size. Another possibility is that stenting may trigger acute vasoconstriction by its effect on microvascular endothelium and result in the use of the smaller sized stent. We could explain the second situation as follows: Either retracting the deflated stent balloon quickly with maximum force or not checking the deflating balloon might result in trapping of the stent balloon by the proximal stent. Also, 24-hour time is early for stent endothelialization and mechanical effects during stent balloon retrieval may lead to stent dislodgement.

Retrieval methods in fully expanded stents have several limitations. Only a few cases have been reported and it was showed that the complete deployed stent was successfully removed with the twisted wire method in a case $^{(11)}$. However, we could not apply this method due to catheter-related vasospasm and we successfully mounted this dislodged stent into the coronary vessel by using crushing technique. Control angiography was performed because the crushing method might increase the risk of restenosis especially in the use of bare metal stents and critical lesions ${ }^{(3)}$. As in our case, the use of crushing method for stent dislodgement was reported in a 47-year-old male patient with unstable angina and in a 59-year-old woman with acute STEMI because of the failure of retrieval methods and similarly, no complications developed during these procedures. However, in these cases, unlike ours, only inflated balloon was used for crushing technique and this method was applied to dislodged unexpanded stents $^{(14,15)}$.

In conclusion, stent dislodgement is a rare but serious complication, so extra care should be given. Crushing method can be used rather than retrieval methods due to their limited use in fully expanded stents. The strategies used to deal with this complication may differ among patients. Therefore, it should be noted that the use of different equipment or several techniques can be combined for various scenarios.

\section{Ethics}

Informed Consent: Informed consent was obtained from the patient.

Peer-review: Externally peer-reviewed. 


\section{Authorship Contributions}

Surgical and Medical Practices: I.T., S.Ç.Ş., Concept: S.Ç.Ş., I.T., Design: S.Ç.Ş., Data Collection or Processing: S.Ç.Ş., M.Ş., Analysis or Interpretation: S.Ç.Ş., Literature Search: S.Ç.Ş., Writing: S.Ç.Ş.

Conflict of Interest: No conflict of interest was declared by the authors.

Financial Disclosure: The authors declared that this study received no financial support.

\section{References}

1. Sentürk T, Ozdemir B, Yeşilbursa D, Serdar OA. Dislodgement of a sirolimus-eluting stent in the circumflex artery and its successful deployment with a small-balloon technique. Turk Kardiyol Dern Ars 2011;39:418-21.

2. Fischman DL, Leon MB, Baim DS, et al. A randomized comparison of coronary-stent placement and balloon angioplasty in the treatment of coronary artery disease. Stent Restenosis Study Investigators. N Engl J Med 1994;331:496-501.

3. Brikalis ES, Best PJM, Elesber AA, et al. Incidence, retrieval methods and outcomes of stent loss during percutaneous coronary intervention: a large single-center experience. Catheter Cardiovasc Interv 2005;66:333-40.

4. Yang Soon C, Chong E, Sangiorgi GM. A challenging case of dislodged stent retrieval with the use of Goose neck snare kit. Catheter Cardiovasc Interv 2010;75:630-3.

5. Bolte J, Neumann U, Pfafferott C, et al. Incidence, management, and outcome of stent loss during intracoronary stenting. Am J Cardiol 2001;88:565-7.
6. Gan HW, Bhasin A, Wu CJ. Complete stent dislodgement after successful implantation-A rare case. Catheter Cardiovasc Interv 2010;75:967-70.

7. Alomar ME, Michael TT, Patel VG, et al. Stent loss and retrieval during percutaneous coronary interventions: a systematic review and metaanalysis. J Invasive Cardiol 2013;25:637-41.

8. Sinha SK, Razi M, Thakur R, et al. Acute dislocation of fully deployed stent after use of non-compliant balloon: an enigma. Folia Cardiologica 2016;11:222-5.

9. Degirmenci H, Bakırcı EM. Approach to coronary stent stripping. MN Cardiology 2018;25:87-92.

10. Esenboğa K. The stent was wriggled out in left main coronary: we tried many things and at the end retrieved it by snare. Van Med Journal 2018;25:253-6.

11. Hsu PC, Lin TH, Lee WH, Sheu SH. Inadvertent extraction of a deployed stent after using twisted wire technique. Kaohsiung J Med Sci 2014;30:556.

12. Hwang J, Chun KJ, Lee DS, et al. Extraction of a Fully Deployed Coronary Stent during Retrieval of Another Dislodged Stent. Korean Circ J 2016;46:862-5.

13. Porwal SC, Halkati PC, Patted SV, Joshi A. Successful deployment of a dislodged sirolimus- eluting stent with a small-balloon technique. J Cardiol Cases 2013;8:155-7

14. Wongpraparut N, Yalamachili V, Leesar MA. Novel implication of combined stent crsushing and intravascular ultrasound for dislodged stents. J Invasive Cardiol 2004; 16:445-6.

15. Stajic Z. Stent dislodgement in the distal left main coronary artery and its successful management with balloon crushing technique. Vojnosanit Pregl 2015;72:454-7. 